\title{
Appreciation of the Piano Work Sonatine by Maurice Ravel
}

\author{
Yuan Bo-Xiao ${ }^{1, a}$ \\ ${ }^{1}$ Nanyang Institute of Technology, Nanyang 473004,China \\ a nylgxyybx@126.com
}

Key words: Ravel; Sonatine; melody; playing style;

\begin{abstract}
This paper analyzes the piano work Sonatine by Maurice Ravel in terms of its melody, leading pitch interval, key tone and the material that forms climax. Through the analysis on such playing styles as polyphony imagination, speed explanation, pedal using and the meaning of dynamics, this paper aims to reveal its uniqueness and level so as to explain the relevance with playing. According to the author, the three movements with different characteristics create the different images of music. The interval, tone and figure are the same in minutia feature. While the melodic structure is unified and the playing style is both unique and intertextual.
\end{abstract}

\section{Introduction}

As one of the most excellent impressionist composers, the French composer Maurice Ravel (1875-1937) breaks the boundaries of traditional disciplines in a creative way by adhering to the principles of Viennese Classical School. He greatly develops the expressiveness of impressionist music. Most of his works are describing the worldly possessions, but they are not confined to the deliberate description of program music. Instead, they are based on objective things with high vision. The early piano work of Ravel is Sonatine in 1905. It was the work that Ravel used for competition at the very beginning. However, it received multiple applauses after playing and they are greatly admired by the listeners. Later it was promoted and published, which has become one of the representative works of Ravel. It mixes various skills of impressionist playing, including polyphony, register, dynamics, speed, harmony and pedal. In the harmony, diatonic scale, sekunde and highly accumulated chord are applied. The sound is smooth and clear and the harmony is delicate. In terms of the overall structure, it has inherited the tradition of classicism and uses sonata type. Three music creation carriers are sonata form, ternary form and rondo sonata form. But they are not limited to the traditional structure. Through the breakthrough in such aspects as length adjustment, tempo change and the use of polyrhythm, the structure is concise without being dull. By incorporating the appeal of Spanish music into the rhythm, it is especially exotic. This is a masterpiece of Ravel by combing the classic with impression.

\section{Rhythm This Part Starts With The Rhythm Of The Work.}

The uniqueness of the work is analyzed through such perspectives as the form of rhythm, melodic interval, main melody and climax melody by the analysis on the distribution of rhythm in the work.

The form of melody is the combination of dynamic and static movement, which reflects the external contour of the melody. Through this contour, the player can play the melody.

The soul of the whole movement is the first three bars of the theme in the first movement. The melodic interval is the fourth jump at the beginning, then the progressive upward, at last it is the third movement after reaching the highest pitch. Seen from the partial contour, it is the transformation from ray type into the zigzag type. The whole contour turns into the interrogative band melody. The tense atmosphere of the whole movement is the transformation from dynamic 
into the static, then it turns into the dynamic. The first theme mainly reflects the elegant and charming melody surrounded by cadenza notes. The music and phrase play synchronized role. In playing, it remains the gorgeous notes and fully expresses the exaggeration of phrase so that it can be "sung" expressively. The first theme is continuous and fluent, which requires the harmony and closeness among penetration, arm movement and breath adjustment in playing skills.

The second movement is extremely charming with the elegance of French music. The melodic interval in the whole movement is the fourth and fifth upward jump at the beginning, then the fifth downward jump, at last it is the third upward jump. It reflects the transformation from ray type to zigzag type then to the ray type in partial contour. It creates the contour of centripetal-centrifuge-centripetal and finishes the change in tension from dynamic to static to dynamic. The playing skill is more difficult, which requires the playing of small sentence with the right hand and interior sound at the same time in the playing method of half jumping and half connecting. The fingers are more often used than pedals so as to produce transparent sound level. In order to play extremely elegant work, the player must pay special attention to the music reading that indicates the change of stress in rhythm.

In the first theme of the third movement, the fourth, fifth and sixth are jumped alternately. The ray type contour outlines stepped dot melody, which describes the dynamic tension. In comparison with it, the fourth of the second theme jumps downward then continuously jumps upward. While the partial ray type presents the centrifuge-centripetal in the whole and the tension transforms from dynamic into static. The melody in the third movement is sometimes static as the sound of the sky and sometimes dynamic as the waterfall among mountains. With its rich music images, abundant colors and great difference, it is extremely fascinating, which requires the player to practice according to the difficult parts and the phases and change the way of touching when necessary.

Melodic interval is seen throughout the work, which reflects the high connotation of melody and plays an important role in forming the main material. In the melodic analysis, "leading pitch interval” is regarded as the backward interval. All the intervals in the main melody of the first movement also can be found in the second and third movements for many times with some change to a certain extent. For example, in the upward jumping interval of the fourth and fifth, the first bar in the first movement for the first time also appears continuously in the 1-12 bar of the first theme in the second movement. It also appears in the 13-22 bar of the second theme and becomes the leading bar in the third movement. The dynamic characteristic is the continuous expanding of interval so as to personalize it to provide it with vigor. Therefore, the player doesn't only need to understand the relevance among all themes of the movements, but also get close to feel the characteristic of the music image in every movement.

The core tone in melodic material decides the characteristic of pitch interval and direction in melody to make it appear in the work repeatedly so as to become the key tone. For example, in the eleventh bar of the first movement, at the beginning is the third downward tone, later it is the burst of melodive decomposed by rapid chord. In the short climax of the first theme, the melody is featured by " the surface of harmony". The tone is composed of progressive and small jump. The direction of interval is straight downward. It appears continuously in the 18-20 bar of the first movement, which renders the dreamlike sound effect. It becomes the background music in the third movement. The representation change is as long as 60-94 bars, 25 bars in total. This lays the foundation of its role as the key tone.

As for the repeated occurrence of the similar melodic tone, how to offer it stronger meaning so as to avoid the dull repetition? This requires the player to have a good control of tone in playing. The various main tones in the first movement also change in different parts in the third movement. This 
requires the player to have both the fast playing speed and the exact tone in playing.

The application of melodic material in climax. During the formation of melody, the purpose and logic could reflect the climax melody. In the music, climax melody is divided into three phases, including incubation, burst and recovery. In order to present and grasp the climax more precisely, the player must understand the formation of climax of the music works in a thorough way.

In the climax of the $55^{\text {th }}$ bar of the first movement, the incubation phase is to repeat the melody and strengthen it gradually. The method in climax is still repetition. The continuous floating rhythm intensifies the climax and in the end it is finished by broadening the rhythm. In the second and third movements, this method of climax is inherited and developed to a certain extent.

In the second movement, the different registers of a phrase is advanced in the wave style to reach the climax. Then through the method of repetitive melody and the use of dotted rhythm throughout the work, the climax gains great momentum and becomes more passionate. After the climax, by reducing the sound of melody and playing downward Pipa, the work creates a quite atmosphere so as to lay the groundwork for the melody of the next music.

In the $54^{\text {th }}, 72^{\text {nd }}$ and $135^{\text {th }}$ bars of the third movement, there are regional climaxes, among which after a longer progressive direction, the climax of the $54^{\text {th }}$ bar appears. While the climaxes in the last two parts are very sudden with shorter direction and quick downward recovery. All of these climaxes are the quantitative accumulations of the momentum development and change of the power of the whole work. The appearance of the whole climax is quite natural, which makes the purpose and logic of the melody even more precise. The climax begins at the $150^{\text {th }}$ bar and sustains until the end of it. There are 7 preparations of the bar in the incubation phase. The material with the most dynamic characteristic in the second theme is quite. It appears repeatedly in different parts and registers. In the end, it finishes the whole work with strong power.

In the works of Ravel, there are often three phrases of the repeated melody. This requires the player to gradually grasp the extent in playing and increasingly intensify so as to be prepared for the climax. There is long end rhythm before the end of the playing. This requires the player to be very attentive when playing. Because the player needs to constantly change the tone in very short time. And the player also need to strengthen the power of music and coordinate all parts of the body in playing. Therefore, the player needs to keep practicing and improve the coordination.

In terms of the melodic structure and music image of the Sonatine, the three movements have different characteristics and music images. Viewed from such factors that compose the rhythm as the pitch interval, tone and figure, they have the characteristics of the same minutia feature and the unified melodic structure. A musician must understand the music from two levels: One is the grasp on the connotation of the work. The other is the grasp on the feel of the work. This requires the player to understand the musician work from all perspectives and know the logic of the work.

Playing style The player can observe the creation intention and expression of the feeling of a work through analysis on the musical work. Through the rational analysis on the elements in the first movement and the practical grasp of the playing style, this paper aims to reveal the unique playing style of the work so as to explain the intertextuality of the playing style.

When playing, it is possible and necessary to draw more from the imagination of the band. Sonatine was once adapted into the flute, cello and harp by Carlos Salzedo. The demisemiquaver and semiquaver are very flexible with some jumps. This requires the player to have high command of the fingers and play it as clearly as the harp. The stable playing of semiquaver in the third part could fill the first beat of the second part and present the whole music with better beat. The fourth tremble needs to be finished without stop in playing. The elegant rhythm in the first part should be played with flute, which could not only reflect the vivid atmosphere but also create a sense of 
reserve. The cello is played in the third part. By using pedal and playing with different touchpad, the rhythm is even more delicate. It is better to combine the high and low sound. When playing in the touchpad, one need to slow down. Through the power at fingertips, the player connects the two outer parts to the largest extent. In this way, the music is both lively and elegant like the gentle telling of the flute.

In the working out part of the 43-58 bars, the polyphony gets thicker to advance the music, which indicates the gradual application of the musical instruments. This could raise the power of register and density of notes to a large extent. There is no big change in the whole music. But the power in 46, 48, 52 bars is stronger, which is caused by the change of musical instruments. This phrase is produced by the change from left hand to right hand, which makes the sound even more beautiful. Through the imagination of band and ensemble, the player could better present the various change of colors in the individual piano. The proper polyphony could help player to better play the music style of Sonatine.

Speed explanation originates from music. But there are not many connections between the speed mark and the content of music when the speed and result it needs could be reflected from the content of music. From the band speed of Ravel, it can be summarized as lively and elegant. Perlemuter said that as for the speed, Ravel asks simple playing without expansion and joint treatment. As for the subtitle, he attaches importance to the preciseness of semiquaver. The octave under the subtitle should not be played too compassionately. If it is played with too weakly, it will lose the rhythm Ravel asks. While Jourdin Morand said that for the works of Ravel, the biggest difficulty is to play both gently and clearly in the "expansion and joint treatment”. Cardo Venace told me that it should be avoided to highlight the first beat to make it vulgar.

In the application of pedals, the theme and power of playing should be strictly stressed. For example, the stress and sound could use the pedal to strengthen the effect of playing. As for the theme in subtitle, it could also use the pedal to make the line of music more coherent and more stable in playing. In the end of the theme, the functional harmony could be used as the main body. The non-harmonic tone becomes the Pipa playing of the seventh, ninth, eleventh and even the thirteenth chord. In applying the pedal, the player should have sensitive control of the foot. Every chord should be transformed clearly. At the same time, the pedal should be connected with the harmony so as to create the atmosphere to control the music to realize the rich harmony colors and give more time and space to the music. In this way, the audience will have more expectation on the next theme. This fully demonstrates the combination of inheriting and breaking the tradition of Ravel. This well known orchestral master creates the wonderful and glorious movement effect.

Meaning of dynamic. In the brief working out part of the movement, the music begins to extend the question. It explores and seeks new expectation. The method of harmony is variously delicate with the harmonies effect of the impressionist. In the 33 bar, the music before the main body remains weak, which is like the darkness before the dawn. While the appearance of the theme is like the first sunshine in darkness shining on the ground. This is exactly where hope begins. The dynamic mark of the music is not only a good sound design for player, but also the confirmation of the structure of the work. In the recapitulation, the theme of the main body is once again reflected. The composer marks mp. In comparison with the exposition, the music is warm and filled with infinite imagination of the future. The work finally ends in the ninth chord that repeats three times.

\section{Conclusion}

With his unique rebellion spirit, Ravel combines the traditional impressionist writing and classical music writing and he puts forward many innovations. This paper analyzes Sonatine, the 
representative piano work of Ravel from two aspects, including melody and playing style. Three movements with different characteristics create different music images. The interval, tone and figure are the same in minutia feature. While the melodic structure is unified and the playing style is both unique and intertextual.

\section{References}

[1] Gao Xiaoguang and Wu Guozhu. Encyclopedia for the art of piano [Z]. Beijing: Encyclopedia of China Publishing House, 2001: 488.

[2] Peng Zhimin. Basic course on musical analysis [M]. Beijing: People’s Music Publishing House, 1997: 68-72.

[3] Yang Ruhuai. The analysis and creation of music (1) [Z]. Beijing: People’s Music Publishing House, 1995: 64.

[4] Yu Runyang. New version of the history on music [M]. Beijing: People's Music Publishing House, 2003: 81. 